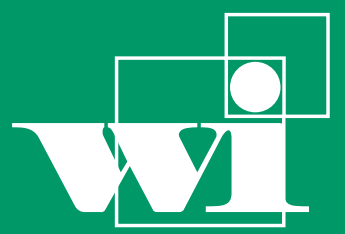

Bayreuther Arbeitspapiere zur Wirtschaftsinformatik

Armin Heinzl

Erkenntnisziele der Wirtschaftsinformatik in den nächsten drei und zehn Jahren

Bayreuth Reports on Information Systems Management 
Die Arbeitspapiere des Lehrstuhls für Wirtschaftsinformatik dienen der Darstellung vorläufiger Ergebnisse, die i. d. R. noch für spätere Veröffentlichungen überarbeitet werden. Die Autoren sind deshalb für kritische Hinweise dankbar.

Alle Rechte vorbehalten. Insbesondere die der Übersetzung, des Nachdruckes, des Vortrags, der Entnahme von Abbildungen und Tabellen auch bei nur auszugsweiser Verwertung.

Authors:

Armin Heinzl
The Bayreuth Reports on Information Systems Management comprise preliminary results which will usually be revised for subsequent publications. Critical comments would be appreciated by the authors.

All rights reserved. No part of this report may be reproduced by any means, or translated.

\section{Information Systems Management Working Paper Series}

\section{Edited by:}

Prof. Dr. Armin Heinzl

\section{Managing Assistant and Contact:}

Universität Bayreuth

Lehrstuhl für Wirtschaftsinformatik (BWL VII)

Prof. Dr. Torsten Eymann

Universitätsstrasse 30

95447 Bayreuth

Germany

Email: wi@uni-bayreuth.de
ISSN $1864-9300$ 


\title{
Erkenntnisziele der Wirtschaftsinformatik in den nächsten drei und zehn Jahren
}

\author{
Prof. Dr. Armin Heinzl \\ Lehrstuhl für Wirtschaftsinformatik \\ Universität Bayreuth \\ Universitätsstraße 30 \\ D-95447 Bayreuth \\ heinzl@uni-bayreuth.de \\ Prof. Dr. Wolfgang König \\ Institut für Wirtschaftsinformatik \\ Universität Frankfurt \\ Mertonstr. 17 \\ D 60054 Frankfurt a. M. \\ wkoenig@wiwi.uni-frankfurt.de \\ Dipl. Kfm. Joachim Hack
Institut für Wirtschaftsinformatik
Universität Frankfurt
Mertonstr. 17
60054 Frankfurt a. M.
hack@wiwi.uni-frankfurt.de

\section{Arbeitspapiere Wirtschaftsinformatik}

Herausgeber: Prof. Dr. Armin Heinzl 


\title{
Zusammenfassung
}

Fünf Jahre nach einer ersten entsprechenden Untersuchung wurde 1999 eine Delphi-Studie durchgeführt, die nach den zentralen Erkenntniszielen der Wirtschaftsinformatik in einem Drei- und Zehnjahreszeitraum fragte, damit diese sich im Wettbewerb mit den Nachbardisziplinen Wirtschaftswissenschaften und Informatik behaupten kann. Der vorliegende Beitrag beschreibt den Hintergrund dieser Arbeit, die zu Grunde liegende Methodik sowie die wichtigsten Ergebnisse, auch im Vergleich zu den Resultaten aus dem Jahr 1994.

\begin{abstract}
Which future research subjects shall be persued by the German speaking IS community (Wirtschaftsinformatik) in order to maintain competitive against other disciplines like business administration and computer science? This article summarizes the major results of a Delphi study which was conducted in 1999 in order to answer this question drawing on the expertise of leading academics and practitioners in the field. Thus, the foundations of this study will be outlined as well as its methodology, the main findings, and a comparison to a similar study which was conducted in 1994.
\end{abstract}

\section{Englischsprachiger Titel}

Future Research Subjects of the German speaking IS Community: a Three Year and Ten Year Perspective

\section{Stichworte}

Erkenntnisziele, Wirtschaftsinformatik, Wirtschaftswissenschaften, Informatik, Delphi

\section{Key words}

Research subjects, Information Systems, Business Administration, Computer Science, Delphi 


\section{Kernpunkte für das Management}

- Für den Zeitraum der nächsten drei Jahre haben 30 Experten die Schaffung verbesserten Wissens über

- Netzmärkte und E-Commerce,

- die Architektur von Informations- und Kommunikationssystemen sowie

- den Zusammenhang zwischen Informationstechnologien und Organisation als bedeutendste Erkenntnisziele formuliert.

- Im Zehnjahreszeitraum wird der Schaffung verbesserten Wissens über

- die Beherrschung von Komplexität in Informations- und Kommunikationssystemen,

- Netzmärkte und virtuelle Märkte sowie

- die Gestaltung der Anwender- bzw. Mensch/Maschine-Schnittstelle die größte Bedeutung beigemessen.

- Die Schaffung verbesserten Wissens über Grundlagen der Wirtschaftsinformatik sowie über Schnittstellen zu anderen Wissenschaften landen in beiden Prognosezeiträumen auf den letzten Plätzen.

- Das Panel sieht die Anwendbarkeit von Forschungsergebnissen in der Praxis als wichtigste Einflussgröße für die Beurteilung von Erkenntniszielen der Wirtschaftsinformatik im Wettbewerbskontext zu den Nachbardisziplinen an.

- $\quad 90 \%$ der Experten sehen das interdisziplinäre Arbeiten als eine Kernkompetenz der Wirtschaftsinformatik und fast drei Viertel von ihnen plädieren für eine Vertiefung der interdisziplinären Bezüge zwischen den Wirtschaftswissenschaften und der Informatik. 


\section{Erkenntnisziele der Wirtschaftsinformatik in den nächsten drei und zehn Jahren}

Armin Heinzl, Wolfgang König und Joachim Hack

\section{Einleitung}

Mitte der neunziger Jahre wurde erstmals untersucht, welchen Forschungsgegenständen sich die Wirtschaftsinformatik als eigenständige Disziplin widmen soll, um im Wettbewerb gegenüber Betriebswirtschaftslehre und Informatik bestehen zu können [KHP95; KHRP96]. Seither sind eine Reihe von Ereignissen und Entwicklungen eingetreten, die darauf schließen lassen, dass die Wettbewerbsintensität weiter zugenommen hat. Folgende Punkte veranschaulichen dies im Kontext knapper werdender öffentlicher Mittel beispielhaft:

- Die Errichtung neuer Studiengänge und Lehrstühle in der Wirtschaftsinformatik stagnierte in den fünf Jahren vor dem Einsetzen der Diskussionen um die „Green Card“.

- Die Debatte um den Mangel an IT-Spezialisten findet in der Öffentlichkeit weitgehend ohne Berücksichtigung der Disziplin Wirtschaftsinformatik statt.

- Drei bei der DFG beantragte Schwerpunktprogramme kamen nicht zur Förderung.

- Im Bereich des Electronic Commerce werden Lehrstühle an Wissenschaftler mit ausschließlich betriebswirtschaftlichem Hintergrund vergeben.

Dies ist nach fünf Jahren Grund genug, die Frage nach den zentralen Forschungsgegenständen der Wirtschaftsinformatik, erneut aufzuwerfen. Sie sollen dazu beitragen, die Wettbewerbsposition dieser Disziplin gegenüber ihren Nachbarn zu stärken, Der vorliegende Beitrag behandelt die Ergebnisse einer dafür durchgeführten Delphi-Studie, an der 199935 Wirtschaftsinformatiker, Wirtschaftswissenschafter, Informatiker und hochrangige Praktiker teilgenommen haben.

Es folgt in Kapitel 2 die Beschreibung der Methodik der Untersuchung. In Kapitel 3 legen wir die Ergebnisse der Forschung dar. Kapitel 4 diskutiert die Untersuchungsergebnisse. Der Beitrag schließt mit einer Zusammenfassung und einem Ausblick (Kapitel 5).

\section{Methodik der Untersuchung}

Zur Untersuchung haben wir - wie im Jahr 1994 - die Delphi-Methode herangezogen. Mit dieser Prognose- und Entscheidungsmethode werden komplexe qualitative Problemstellungen, die nicht als Trend extrapolierbar sind, von einer Gruppe räumlich verteilt agierender Experten (dem sog. Panel) asynchron, anonym und rückkoppelnd über mehrere Runden analysiert und bewertet (vgl. hierzu [RoWr99] sowie [Hein96] und die dort angegebene Literatur). Gegenüber der letzten Studie wurden drei Veränderungen im Untersuchungsansatz vorgenommen:

a) Vor fünf Jahren bezog die Untersuchung neben den Erkenntnisgegenständen auch Erkenntnismethoden und Theoriekerne mit ein. Der Einwand von Panelisten, die Relevanz von Forschungsmethoden der Wirtschaftsinformatik könne nicht unabhängig von den Forschungsgegenständen bewertet werden [KHRP96, 38], hat die Autoren dieser Studie dazu veranlasst, den Schwerpunkt ausschließlich auf Erkenntnisgegenstände zu legen und dieser Argumentation entsprechend auch auf eine Ergründung von Theorienkernen zu verzichten. 
b) In der Studie von 1994 wiesen mehrere Teilnehmer auf den mit zehn Jahren relativ langen Prognosezeitraum hin. Es wurde argumentiert, dass die Entwicklungen in der Informationstechnik zu dynamisch seien, um sie zehn Jahre im Voraus abschätzen zu können. Die vorliegende Delphi-Studie trägt dem Rechnung und unterscheidet mit drei und zehn Jahren zwei Prognosezeiträume im Sinne einer kurz- und langfristigen Ausrichtung der Wirtschaftsinformatik als Wissenschaftsdisziplin.

c) Um deutlicher herauszuarbeiten, dass die Beschäftigung mit Erkenntnisgegenständen kein Selbstzweck ist, sondern die Erarbeitung neuen Wissens die herausragende Zielsetzung einer Wissenschaft ist, tritt in der vorliegenden Studie der Begriff „Erkenntnisziel“ an die Stelle des Terms „Erkenntnisgegenstand“. Das Beispiel „Erarbeitung verbesserten Wissens über Netzmärkte und Electronic Commerce" verdeutlicht, dass es sich hierbei um imperative Erkenntnisgegenstände handelt, die in der vorliegenden Studie semantisch präzisiert werden sollen.

Zur Teilnahme an der Studie wurden 52 hochrangige Persönlichkeiten aus Wissenschaft und Praxis eingeladen. Das primäre Auswahlkriterium war hierbei das Potenzial dieser Experten, die Wirtschaftsinformatik in Wissenschaft und Praxis in den vor uns liegenden Jahren fortzuentwickeln und zu gestalten. 35 der Angesprochenen sagten zu, was einer Quote von 67\% entspricht. Im Verlauf des dreiphasigen Vorgehens lag die Abbruchrate bis zum Abschluss der Runde zwei, deren Ergebnisse im Zentrum der Berichterstattung in Kapitel 3 stehen, bei 9\%, d. h. drei Panelisten. Zwei weitere Experten verließen das Panel nach Runde 2. Eine Liste der 30 Experten, welche bis zum Abschluss der Studie im Panel verblieben, findet sich im Anhang A.

Von den bis Ende der Runde zwei aktiven Teilnehmern sind 18 (56\%) Wirtschaftsinformatiker. Sechs Panelisten (19\%) weisen einen wirtschaftswissenschaftlichen Hintergrund auf (fünf Betriebswirte und ein Volkswirt) und weitere acht (25\%) sind Informatiker. Differenziert man das Panel nach dem Aufgabenschwerpunkt der Teilnehmer, so sind 27 Personen $(84 \%)$ in Forschung und Lehre tätig, während fünf (16\%) einer Beschäftigung in der Wirtschaft nachgehen.

Von dem Angebot, die Fragebögen für die einzelnen Runden im Internet auszufüllen, machten $84 \%$ der Teilnehmer Gebrauch. Dieses Medium bot den Vorteil, dass erstmals alle Antworten der Experten im authentischen Wortlaut zur Verfügung gestellt werden konnten, wenn dies bei der Beantwortung des Fragebogens gewünscht wurde. Die daraus resultierende umfangreiche Informationsmenge wurde mithilfe der Hypertexttechnik hierarchisch strukturiert. Lediglich fünf Teilnehmer (16\%) zogen einen gedruckten und zugeschickten Fragebogen vor. Keiner dieser Experten bediente sich der angebotenen Möglichkeit, solche ergänzenden Dokumente anzufordern, die im Internet durch Hyperlinks zugreifbar waren. Der Delphi-Prozess dauerte drei Runden und wurde von April bis Juli 1999 durchgeführt.

Die Eröffnungsfrage (Runde eins) lautete: „Welche sind die wesentlichen Erkenntnisziele der Wirtschaftsinformatik in den nächsten drei bzw. zehn Jahren, um im Wettbewerb mit den Nachbardisziplinen Betriebswirtschaftslehre und Informatik bestehen zu können“. Damit blieb - analog zu 1994 - das Befragungsformat zunächst offen und es wurde wiederum ein Wettbewerbsmodell zu Grunde gelegt. Die Teilnehmer wurden gebeten, für den Drei- bzw. Zehnjahreszeitraum bis zu drei Erkenntnisziele anzugeben. Das Moderatorenteam sammelte und klassifizierte die Antworten und überführte die von den Teilnehmern formulierten Ziele in ein geschlossenes Format. Die so verdichteten Erkenntnisziele (sog. „Cluster“) wurden in Runde zwei den Panelisten zur Bewertung vorgelegt. Diese erfolgte mithilfe von fünf Kon- 
stituenten, deren jeweiliger Ergebnisbeitrag auf einer fünf Punkte umfassenden Likert-Skala einzuschätzen war (von 1 (sehr niedrig) bis 5 (sehr hoch)). Als Konstituenten kamen zur Anwendung:

- das Ausmaß der einem Erkenntnisziel zuzuordnenden grundlegenden Bedeutung für die Gesellschaft (man kann sich den Stellenwert dieses Beurteilungsfaktors an einem Erkenntnisziel der Medizin „Finden eines Mittels zur Heilung von HIV-Infektionen“ verdeutlichen),

- die Anwendbarkeit der für ein Erkenntnisziel erwarteten Ergebnisse in der Praxis (es sollen solche Aufgabenstellungen, die auf in der Praxis kaum auftretenden Voraussetzungen beruhen oder im Vergleich sehr enge Fragestellungen aufweisen und daher weniger breit zur Anwendung kommen können, erkannt werden),

- das Ausmaß der einem Erkenntnisziel innewohnenden Interdisziplinarität, d. h. die Anzahl der zur Erreichung des Erkenntnisziels einzubeziehenden Disziplinen und deren Unterschiedlichkeit,

- die beim Einsatz der notwendigen Methoden zur Erarbeitung der Ergebnisse zu erwartende Allgemeingültigkeit der Aussagen sowie

- die erwartete Reproduzierbarkeit von Ergebnissen.

Die beiden erstgenannten Konstituenten adressieren sachliche Gütekriterien der Erkenntnisziele, während die beiden am Schluss positionierten die Qualität der zu einem Ziel passenden Methoden behandeln. Das Beurteilungskriterium Interdisziplinarität ist sowohl der sachlichen als auch der methodischen Dimension zuzurechnen. In Runde eins hatten wir jeden Teilnehmer gebeten, eine individuelle Gewichtung der Konstituenten vorzunehmen. Anhand der Gewichte und der jeweiligen Bewertungen wurde für jedes Cluster pro Panelist ein additiv ermittelter gesamter Nutzwert berechnet [BaCo96, 49 ff.]. Das arithmetische Mittel der Nutzwerte über alle Teilnehmer diente anschließend als Basis für die Bildung einer Rangfolge der Cluster.

In Runde drei wurde den Teilnehmern die Rangfolge der Cluster mit den arithmetischen Mitteln der Nutzwerte und ihrer Standardabweichung als Ausgangsinformation unterbreitet. Zudem erfolgte die Darstellung der eigenen Antwort in Relation zu der aggregierten Antwort des Panels. Bei Erkenntniszielen, bei denen die Einschätzung eines Panelisten außerhalb des mittleren Quartilsabstands lag, wurde der betreffende Teilnehmer gebeten, seine abweichende Einschätzung zu begründen oder seine Bewertung zu überarbeiten. In dieser Runde erstellten wir softwaregestützt für jeden Teilnehmer einen individuellen Fragebogen, sodass dieser sich nur mit seinen drei am höchsten bewerteten Clustern vertieft auseinandersetzen musste, was ebenfalls ein Novum gegenüber der letzten Studie darstellt. Dadurch konnte der Bearbeitungsaufwand der Experten reduziert werden, ohne die Aussagekraft der Studie signifikant einzuschränken. Das vorliegende Datenmaterial birgt keine Hinweise, dass stabile unterschiedliche Meinungen im Panel durch die Mittelwertbildung vereinheitlicht wurden.

Die Moderatoren haben in Verfolgung zahlreicher Anmerkungen und Kommentare in Runde drei auf die Interdisziplinarität als Konstituente zur Nutzwertermittlung verzichtet. Als zentrales Argument wurde von mehreren Teilnehmern angeführt, dass Interdisziplinarität wertneutral und damit kein geeigneter Maßstab zur Beurteilung der Wirtschaftsinformatik als attraktive Wissenschaft ist. Wir übernehmen diese Argumentation im Rahmen dieser Auswertung, d. h., die Konstituente Interdisziplinarität wird bei der Bildung der Rangplätze nicht berücksichtigt. Die Gewichte der verbleibenden vier Konstituenten werden unter Auslassung der Interdisziplinarität auf eins normiert und neu berechnet. Als Datenbasis ziehen wir für diese Konstituenten die Antworten aus Runde zwei heran, da hier die Eigenschaft einer statistisch 
unabhängigen Stichprobe vorliegt [Sack75]. Die Rangfolge der Cluster wurde auf der Basis der gewichteten Teilnutzwerte gebildet. Des Weiteren haben wir Unterschiede zwischen den Mittelwerten mittels T-Tests unter entsprechender Angabe des Signifikanzniveaus $\alpha$ ermittelt.

Für die Auswertung der Antworten zur Interdisziplinarität, die in Runde drei Gegenstand eines eigenständigen Diskussionsteils wurde, haben wir die Daten aus eben dieser Runde herangezogen (siehe Abschnitt 3.3).

\section{Ergebnisse}

Zunächst beleuchten wir die Rezeption des Wettbewerbsmodells seitens der Experten (Abschnitt 3.1) und diskutieren die Bedeutung der Konstituenten (Abschnitt 3.2), gefolgt von Resultaten zur Interdisziplinarität als Kernkompetenz der Wirtschaftsinformatik (Abschnitt 3.3). In Abschnitt 3.4 stellen wir die auf der Basis der verbleibenden vier Konstituentengewichte berechneten Nutzwerte für die in Runde zwei gebildeten Cluster für den Dreijahreszeitraum vor. Die entsprechenden Zehnjahreswerte sind Gegenstand von Abschnitt 3.5. Zum Abschluss dieses Kapitels vergleichen wir die Ergebnisse für die beiden Zeiträume.

\subsection{Diskussion des zu Grunde liegenden Wettbewerbsmodells}

Wenige Panelisten äußern Bedenken hinsichtlich des Wettbewerbsmodells, in dessen Rahmen die folgenden Ausführungen getroffen werden. Eine Vielzahl anderer Experten spricht hingegen wörtlich oder sinngemäß von einem ,sinnvoll empfundenen Wettbewerbskontext“. Ein Mitwirkender führt aus: „Ich vermute, dass in zehn Jahren die strukturellen und institutionellen Ausrichtungsprobleme der Wirtschaftsinformatik dahingehend gelöst sein werden, dass es eigenständige Disziplinen wie Electronic Commerce (entsprechende Studiengänge werden ja bereits eingerichtet) geben wird. Die Frage ist, was dann noch von der heutigen Wirtschaftsinformatik übrig bleibt.“ Es steht also nicht zur Debatte, ob Wettbewerb besteht, sondern zu welchen (ggf. zusätzlichen) Disziplinen er stattfindet. Darüber hinaus lehrt die Praxis, dass zwischen Akteuren gleichzeitig Wettbewerb und Kooperation stattfinden kann [NaBr99], worauf ein Teilnehmer hinweist.

\subsection{Relative Bedeutung der Konstituenten}

Die Experten messen der Konstituente „Anwendbarkeit in der Praxis“ mit 30\% ( $\overline{\mathbf{X}}=0,30)$ die höchste relative Bedeutung zu, wobei die Vertreter der Wirtschaft im Panel diesen Faktor mit $37 \%$ gewichten. Hier spiegelt sich der starke Anwendungsbezug der Wirtschaftsinformatik wieder. Am Zweitwichtigsten wird das „Ausmaß einer grundlegenden Bedeutung von Erkenntniszielen für die Gesellschaft“ eingestuft $(\bar{x}=0,26)$, gefolgt von der „Allgemeingültigkeit der Aussagen“ $(\overline{\mathbf{x}}=0,22)$ sowie der „Reproduzierbarkeit von Ergebnissen“ $(\overline{\mathbf{x}}=0,21)$. Damit wird die inhaltliche Relevanz von Erkenntniszielen wichtiger eingestuft als die mit ihrer Erarbeitung verbundene methodische Stringenz. Dies zeigt auch ein beispielhafter Kommentar: „Gerade unter dem Gesichtspunkt der Wettbewerbsposition [...] kommen den Punkten „Bedeutung für die Gesellschaft“ und „Praxisrelevanz“ entscheidende Bedeutung zu.“"Ein anderer Teilnehmer ergänzt, dass es um die in der Öffentlichkeit wahrnehmbaren Konstituenten geht, und dies sind in seinen Augen die ersten beiden.

Ein Wirtschaftswissenschaftler, der die „Allgemeingültigkeit der Aussagen“ im Gegensatz zum Mittelwert stärker betont, führt aus: „Der hohe Wert [...] ergibt sich [...] aus der Beo- 
bachtung, dass Wirtschaftsinformatik-Arbeiten - zumindest soweit sie finanzwirtschaftliche Fragen berühren - oft und zu schnell auf spezielle Anwendungsfälle eingehen, ohne nach allgemeinen Zusammenhängen und Gültigkeiten zu fragen." Ein anderer Experte, Wirtschaftsinformatiker, der die Reproduzierbarkeit der Ergebnisse für im Vergleich wichtiger erachtet, formuliert: „Reproduzierbarkeit? Solche Arbeiten kenne ich in der Wirtschaftsinformatik überhaupt nicht.“

Im Rahmen der Diskussion der Konstituenten einer Wissenschaft, über deren grundsätzliche Gültigkeit mit Ausnahme der „Interdisziplinarität“ weitgehend Einigkeit im Panel herrscht, entwickelt sich eine Diskussion über mögliche Klassifizierungen von Wissenschaften und die Einordnung der Wirtschaftsinformatik, die in folgender Frage eines Informatikers zusammengefasst wird: „Analytische Wissenschaften schaffen neue Einsichten in Vorhandenes. Konstruktive Wissenschaften (Ingenieurwissenschaften, BWL) schaffen neue Artefakte (Produkte, Prozesse). Ich frage mich, auf welcher Seite sieht sich eigentlich die Wirtschaftsinformatik?“” Abgesehen von der Notwendigkeit, dass auch in konstruktiven Wissenschaften eine Einsicht in Vorhandenes verfügbar sein sollte, legt das Zahlenmaterial nahe, dass das Panel die Wirtschaftsinformatik eher als konstruktive Wissenschaft ansieht.

In diesem Zusammenhang wird auch ein Disput ausgeführt, welche Komplexitätseigenschaften gegeben sein müssen, um grundsätzlich von einer Wissenschaft zu sprechen. Während mehrere Experten die Meinung vertreten, dass eine anspruchsvolle Methodik vonnöten sei, argumentieren zwei andere Teilnehmer: „Diese Argumentation läuft dann auf die Mathematik als Königin der Wissenschaft hinaus. Wir sollten nicht über den vermeintlichen Schwierigkeitsgrad in Wissenschaftsimperialismus verfallen.“ „Wenn jemand Schwierigkeitsgrad sucht, soll er Schach spielen. Generalisierung von bisher nicht erkannten Gleichheiten, Verbindungen von Einzelaussagen und gerade die Vereinfachung bzw. Einfachheit von Lösungen sind faszinierend.“

Insgesamt sind die quantitativen Bewertungsunterschiede begrenzt und haben sich von Runde zwei zu Runde drei verringert.

\subsection{Interdisziplinarität als Kernkompetenz der Wirtschaftsinformatik}

In Runde eins war zunächst gefragt worden, ob interdisziplinäres Arbeiten als eine Kernkompetenz der Wirtschaftsinformatik anzusehen sei. Nach zwei weiteren Runden bejahen $90 \%$ der Teilnehmer diese Frage. Ergänzend und möglicherweise etwas einschränkend zu dieser hohen Zustimmung weisen einige Panelisten darauf hin, dass Interdisziplinarität zwar eine, aber nicht die einzige Kernkompetenz der Wirtschaftsinformatik sei.

Die Experten, die diese Frage verneinen, begründen dies mit einem möglichen Verlust der Eigenständigkeit der Wirtschaftsinformatik. Ein Teilnehmer führt hierzu aus: „Wie kann man eine Disziplin sein, wenn man Interdisziplinarität zur Kernkompetenz erhebt? Man wird bestenfalls zur Metadisziplin und verabschiedet sich damit von jeder unmittelbaren Rolle als Gestalter". Ein anderer bemerkt, dass interdisziplinäres Arbeiten kein Exklusivmerkmal der Wirtschaftsinformatik ist, sondern eine inhärent notwendige Eigenschaft. Interdisziplinarität folgt aus dem Problem und umfasst möglichst alle Konzepte und Verfahren, die zu dessen Lösung erforderlich sind, unabhängig von deren Etikett. Sie kann nicht an sich angestrebt werden, sondern ist ausschließlich problemkontextspezifisch. Ein Wissenschaftler schreibt zur Ablehnung: „Die Wirtschaftsinformatik liefert einen Pfeiler zu der Brücke, die aus der Wirtschaftsinformatik und einem Anwendungsbereich (z. B. der Finanzierung) erzeugt wird." 
Diejenigen Panelisten, welche die Frage nach Interdisziplinarität als Kernkompetenz der Wirtschaftsinformatik mit ,ja“ beantwortet hatten, erhielten zwei Zusatzfragen. Zum einen wurden sie gebeten, auszuführen, ob die Wirtschaftsinformatik ihr interdisziplinäres Profil über die Wirtschaftswissenschaften und die Informatik hinaus auf weitere Disziplinen ausdehnen soll. Diese Frage bejahen 58\% der der ersten Frage ebenfalls zustimmenden Teilnehmer. Als exemplarische Disziplinen nennen sie Psychologie, Soziologie, Ingenieurwissenschaften, Arbeitswissenschaften, Biologie, Systemwissenschaften und die Politik (diese Liste ist nach abnehmender Häufigkeit der Nennung sortiert).

Zudem wurde gefragt, ob die Wirtschaftsinformatik ihre interdisziplinären Bezüge zu den Wirtschaftswissenschaften und zur Informatik vertiefen soll, was $73 \%$ befürworten. Auf die ergänzende Frage, wie dies am besten geschehen soll, verweisen einige Experten auf jeweils gemeinsame Tagungen, Zeitschriften, Publikationen, Forschungsprojekte, Gremien und auch die gemeinsame Auswahl von Problemstellungen. Dabei sollten nicht nur „Sprachelemente der betreffenden Disziplinen“ (wie es ein Teilnehmer formuliert), sondern vor allem deren Methoden übernommen werden.

Die Vertreter der Wirtschaft im Panel urteilen tendenziell stärker praxisorientiert bei Befürwortung der Interdisziplinarität. Ein entsprechender Experte führt hierzu aus: „Anwendbarkeit in der Praxis auf Basis einer gelebten Interdisziplinarität zwischen Informatik und Wirtschaftswissenschaften sind bei weitem die wichtigsten Kriterien aus unserer Sicht. Die Wirtschaftsinformatik sollte in erster Linie nicht versuchen, die Wirtschaft zu verändern.“ Ein weiterer Panelist aus dieser Gruppe sowie ein Vertreter der Wissenschaft betonen die Notwendigkeit der Vertiefung der interdisziplinären Arbeit zwischen der Informatik und den Wirtschaftswissenschaften, um nicht nur verfügbare „Informatik-Erkenntnisse in den Wirtschaftswissenschaften umzusetzen“, sondern auch umgekehrt bestehende wirtschaftswissenschaftliche Probleme durch geeignete Informationstechnik zu lösen und gar die Fortentwicklung der Informationstechnik aus den Anforderungen komplexer Anwendungsaufgaben heraus zu treiben.

\subsection{Erkenntnisziele der Wirtschaftinformatik in den nächsten drei Jahren}

In Tabelle 1 sind die auf der Basis der Konstituentengewichte berechneten Nutzwerte für die in Runde zwei gebildeten Cluster nach absteigendem Mittelwert sortiert aufgeführt.

\begin{tabular}{|l|c|c|c|}
\hline Kurztitel des Cluster $^{1}$ & Mittelwert & $\begin{array}{c}\text { Standard- } \\
\text { abweichung }\end{array}$ & Rang \\
\hline Netzmärkte und E-Commerce & 3,74 &, 60 & 1 \\
\hline $\begin{array}{l}\text { Architektur von Informations- und Kommunikationssys- } \\
\text { temen }\end{array}$ & 3,56 &, 55 & 2 \\
\hline $\begin{array}{l}\text { Zusammenhang zwischen Informationstechnologie und } \\
\text { Organisation }\end{array}$ & 3,52 &, 66 & 3 \\
\hline Information als Produkt & 3,49 &, 67 & 4 \\
\hline Neue Techniken der Wirtschaftsinformatik & 3,46 &, 57 & 5 \\
\hline Wirtschaftlichkeit und Produktivität & 3,41 &, 63 & 6 \\
\hline Strategische Wirkungen von Informationssystemen & 3,39 &, 54 & 7 \\
\hline
\end{tabular}

\footnotetext{
${ }^{1}$ Aus Übersichtsgründen führen wir in den Tabellen nur den Kurztitel an. Eine Liste der vollständigen ClusterTitel findet sich in Anhang B. Im Verlauf der Studie waren diese im World Wide Web abrufbar.
} 


\begin{tabular}{|l|c|c|c|}
\hline Wissensmanagement & 3,39 &, 82 & 7 \\
\hline $\begin{array}{l}\text { Beherrschbarkeit von Komplexität in Informations- und } \\
\text { Kommunikationssystemen }\end{array}$ & 3,32 &, 79 & 9 \\
\hline Neuartige Anwendungssysteme & 3,29 &, 60 & 10 \\
\hline Management des Wandels von Informationssystemen & 3,07 &, 85 & 11 \\
\hline Folgenabschätzung neuer Technologien & 2,95 &, 61 & 12 \\
\hline $\begin{array}{l}\text { Schnittstellen der Wirtschaftsinformatik zu anderen } \\
\text { Wissenschaften }\end{array}$ & 2,74 &, 81 & 13 \\
\hline Grundlagen der Wirtschaftsinformatik & 2,71 &, 73 & 14 \\
\hline
\end{tabular}

Tabelle 1 Erkenntnisziele der Wirtschaftsinformatik in den nächsten drei Jahren

Nachfolgend wollen wir (wie später auch auch im Abschnitt 3.5) die ersten fünf Ränge näher beleuchten. Im Dreijahreszeitraum räumen die Experten dem Erkenntnisziel ,Schaffung verbesserten Wissens über Netzmärkte und Electronic Commerce“ die höchste Bedeutung ein. Dabei wird im Wesentlichen dessen gesamtwirtschaftliche Bedeutung betont. So führen verschiedene Panelisten an, dass dieses Cluster entscheidend für die Leistungsfähigkeit einer Volkswirtschaft im internationalen Wettbewerb ist und die Wirtschaftsinformatik hierzu viel beitragen kann. Analog argumentiert ein Teilnehmer, der einer Verbesserung des Wissens über Netzmärkte und Electronic Commerce eine zentrale Bedeutung für die Gestaltung der Informationsgesellschaft einräumt. Ein anderer Experte hält es für erstrebenswert, gerade in diesem Bereich von der bisher geübten Vorgehensweise der Beschreibung von Fällen zu methodisch leistungsfähigeren Ansätzen zu gelangen. Es wird zu bedenken gegeben, dass eine weitergehende Methoden- und Verfahrenskompetenz zur Erhöhung der Reproduzierbarkeit von Ergebnissen und der Allgemeingültigkeit der Aussagen für dieses Erkenntnisziel wünschenswert wäre. Diese Einschätzungen spiegeln sich auch in den arithmetischen Mittelwerten der Gewichte wieder, die mit $\overline{\mathbf{x}}_{1 \text {,Praxis }}=1,33$ und $\overline{\mathbf{x}}_{1, \text { Bedeutung }}=1,07$ in dem vorliegenden additiven Modell nahezu zwei Drittel der gesamten Gewichtssumme ausdrücken, während $\overline{\mathrm{X}}_{1, \text { Allgemeingültigkeit }}=0,69$ und $\overline{\mathrm{x}}_{1, \text { Reproduzierbarkeit }}=0,64$ signifikant niedriger gewichtet werden ${ }^{2}$.

Auf dem zweiten Rang stufen die Experten die „Schaffung verbesserten Wissens über die Architektur von Informations- und Kommunikationssystemen" ein. Dabei wird besonders der grundlegende und langfristige Charakter von Architekturen betont. Anhand der Aussage eines Teilnehmers lässt sich dies verdeutlichen: „Auf der Suche nach tieferen Werten oder Zielen bzw. spezifischen Methoden der Wirtschaftsinformatik komme ich immer häufiger auf Architekturfragen. Architekturen sind (bzw. sollten) weitgehend unabhängig vom kurzlebigen technischen Entwicklungsstand sein." Ein anderer Experte hält eine Architektur für das, was lange eine allgemeine Gültigkeit besitzt. Ein weiterer Panelist verknüpft mit diesem Cluster wesentliche Teile des Theorie- und Methodenwissens der Wirtschaftsinformatik. Dieser Zusammenhang lässt sich statistisch nachweisen. Das Cluster „Architektur von Informationsund Kommunikationssystemen“ korreliert signifikant mit dem Cluster „Neue WI-Techniken“ (Rang fünf), auf das nachfolgend noch eingegangen wird.

Über das Erkenntnisziel „Schaffung verbesserten Wissens über den Zusammenhang zwischen Informationstechnologien und Organisation“, das auf den dritten Rang eingestuft wird, entwickeln sich heterogene Bewertungen. Während ein Teilnehmer im Panel davon ausgeht, dass

\footnotetext{
$2 \overline{\mathrm{X}}_{\mathrm{a}, \mathrm{b}}$ bezeichnet den Mittelwert für den Cluster auf Rangplatz a und die Konstituente b. a kann einen ganzzahligen Wert zwischen 1 und 14 annehmen. b bezeichnet eine der vier Konstituenten entsprechend den Ausführungen in Kapitel 2, wobei wir hier wiederum Kurzformen zur Identifikation verwenden. „Bedeutung“ heißt „Ausmaß der einem Erkenntnisziel zuzuordnenden grundlegenden Bedeutung für die Gesellschaft“. Die drei anderen Kurzbezeichnungen für Konstituenten sind selbsterklärend.
} 
die in diesem Kontext getätigten Aussagen viel zu allgemein sind, wundert sich ein anderer Experte über die (niedrige) Einschätzung des Panels. Er sieht in dem vorliegenden Cluster die ureigene Domäne der Wirtschaftsinformatik, in der die Organisation und das Zusammenspiel der beiden Aufgabenträger Mensch und Maschine zur bestmöglichen Erfüllung betrieblicher Aufgaben betrachtet wird. Ein wiederum anderer Teilnehmer fügt hinzu, dass sich das Zusammenspiel von Informationstechnologien und Organisation im Zentrum der Wirtschaftsinformatik befindet, wenn diese Disziplin eine grundlegende Bedeutung für die Gesellschaft besitzt. Konzepte der Wirtschaftsinformatik werden dann zu einem gewissen Grad Abläufe und Organisationsformen in der Wirtschaft verändern. Dieser Teilnehmer geht davon aus, dass reproduzierbaren Ergebnissen aus Fallstudien und Simulationen im Zeitraum von drei Jahren langfristig eine Theoriebildung folgend wird ${ }^{3}$.

Der Cluster „Schaffung verbesserten Wissens über Information als Produkt“ befindet sich auf dem vierten Rang. Information wird als Kern einer Informations- und Wissensgesellschaft angesehen und ihrer Produktion eine herausragende Rolle beigemessen. Verschiedene Teilnehmer verweisen dabei auf den grundlegenden und interdisziplinären Charakter der Arbeiten zur Erreichung dieses Erkenntnisziels. Gegen eine höhere Bedeutung dieses Clusters wird angeführt, dass sich Information als Produkt im Internet nicht durchsetzen wird, da Information immer häufiger als freies Gut verfügbar ist. Ein anderer Teilnehmer ist der Auffassung, dass dieses Thema bereits von der Informationswissenschaft facettenreich bearbeitet worden ist.

Auf dem fünften Rang wird die „Schaffung verbesserten Wissens über neue Techniken der Wirtschaftsinformatik“ eingestuft. Ein Panelist führt hierzu an, dass das Wissen über die Fähigkeiten der Wirtschafsinformatik in der Gesellschaft verankert werden muss, wenn sich diese Disziplin bleibend behaupten will. Das in diesem Zusammenhang erworbene Wissen muss sich zudem durch eine hohe Anwendbarkeit in der Praxis auszeichnen, sonst leidet die Glaubwürdigkeit der Disziplin. Ein anderer Experte merkt allerdings an, dass er beim gegenwärtigen Verhältnis von theoriebasierten Arbeiten und Fallstudien keine hohe Bedeutung erkennen kann.

\subsection{Erkenntnisziele der Wirtschaftsinformatik in den nächsten zehn Jahren}

Die Experten schätzen bei der Analyse von Erkenntniszielen über einen Zeitraum von zehn Jahren die „Schaffung verbesserten Wissens über die Beherrschung von Komplexität in Informations- und Kommunikationssystemen" am höchsten ein (siehe Tabelle 2). Ein Teilnehmer formuliert hierzu die Frage: ,Wenn wir die Komplexität der IuK-Systeme weder beherrschen, noch verstehen, noch akzeptieren, was machen diese Systeme dann mit uns?“. In eine ähnliche Richtung geht der Kommentar eines weiteren Experten, der argumentiert, dass man sich der Komplexität nicht entziehen kann und dies mit der Metapher eines Kaugummis verdeutlicht, der am Finger klebt und nicht zu entfernen ist. Seiner bildhaften Darstellung zufolge mag man sich nur aussuchen, an welchem „Finger“" (diese stehen etwa für Modellierung, Implementierung, Referenzmodell) der Kaugummi am besten beherrschbar ist.

\begin{tabular}{|l|c|c|c|}
\hline${\text { Kurztitel der } \text { Cluster }^{4}}^{\text {Mittel- }}$ & $\begin{array}{c}\text { Standard- } \\
\text { wert } \\
\text { abwei- }\end{array}$ & Rang \\
\hline
\end{tabular}

\footnotetext{
${ }^{3}$ Wobei das vorliegende Datenmaterial diesen Zusammenhang nicht zu bestätigen vermag.

${ }^{4}$ Aus Übersichtsgründen führen wir in den Tabellen nur den Kurztitel an. Eine Liste der vollständigen Cluster-

Titel findet sich in Anhang B. Im Verlauf der Studie waren diese im World Wide Web abrufbar.
} 


\begin{tabular}{|c|c|c|c|}
\hline & & chung & \\
\hline $\begin{array}{l}\text { Beherrschung von Komplexität in Informations- und Kommu- } \\
\text { nikationssystemen }\end{array}$ & 3,70 & ,72 & 1 \\
\hline Netzmärkte und virtuelle Märkte & 3,69 & ,58 & 2 \\
\hline Anwender- / Mensch-Maschine-Schnittstellen & 3,55 & ,64 & 3 \\
\hline Informationsmanagement / Wissensmanagement & 3,47 &, 78 & 4 \\
\hline Architektur von Informationssystemen & 3,45 & ,63 & 5 \\
\hline Neue Arbeitsteilungen und Formen von Kollaborationen & 3,44 & ,78 & 6 \\
\hline Neue Lehr- und Lernformen & 3,33 & ,63 & 7 \\
\hline Künstliche Intelligenz-Systeme / menschenähnliche Systeme & 3,11 & 65 & 8 \\
\hline $\begin{array}{l}\text { Gesellschaftliche Folgen des Einsatzes von Informationssys- } \\
\text { temen }\end{array}$ & 2,97 & ,77 & 9 \\
\hline $\begin{array}{l}\text { Wertorientierte Unternehmensführung und Beitrag der Infor- } \\
\text { mations- und Kommunikationssysteme }\end{array}$ & 2,95 & ,79 & 10 \\
\hline $\begin{array}{l}\text { Human Ressource Management in der Informationstechnolo- } \\
\text { gie }\end{array}$ & 2,89 &, 70 & 11 \\
\hline $\begin{array}{l}\text { Management des Wandels von Informationssystemen sowie } \\
\text { anderer Objekte }\end{array}$ & 2,88 & ,79 & 12 \\
\hline Grundlagen der Wirtschaftsinformatik & 2,85 & ,86 & 13 \\
\hline Neuartige Anwendungssysteme & 2,85 &, 70 & 13 \\
\hline Schnittstellen der Wirtschaftsinformatik & 2,82 & ,95 & 15 \\
\hline
\end{tabular}

Tabelle 2 Erkenntnisziele der Wirtschaftsinformatik in den nächsten zehn Jahren

Die Experten stufen das Erkenntnisziel „Schaffung verbesserten Wissens über Netzmärkte und virtuelle Märkte" auf Platz zwei in statistischer Hinsicht kaum weniger bedeutsam ein. Interessanterweise wird bei diesem Cluster jedoch keine tiefergehende Diskussion geführt, was darauf hindeutet, dass im Wesentlichen die Argumente des kongruenten Erkenntnisziels aus dem Dreijahreszeitraum bei der Beurteilung herangezogen wurden.

Auf dem dritten Rang gelangt die „Schaffung verbesserten Wissens über Anwender- / Mensch-Maschine-Schnittstellen“. Als Hauptgrund für die hohe Einstufung wird angeführt, dass die Effektivität von Informations- und Kommunikationssystemen in der Praxis maßgeblich über die Anwenderschnittstelle bestimmt wird und in besonderem Maße die Systemakzeptanz beeinflusst. Ein anderer Teilnehmer verdeutlicht dies an folgendem Beispiel: „Die Akzeptanz von neuen Medien ist ein zentraler Faktor für die Bedeutung dieser Techniken in der Gesellschaft. Da Telefon, Fax und TV so leicht zu benutzen sind, haben sie sich in der Bevölkerung durchgesetzt und die Gesellschaft entsprechend verändert." Ein anderer Panlist entgegnet, dass die hier bestehenden Probleme schon früher als in zehn Jahren gelöst sein müssen.

An die vierte Stelle setzen die Experten das Erkenntnisziel „Schaffung verbesserten Wissens über Informations- und Wissensmanagement". Ein Teilnehmer führt hierzu aus, dass wir in Daten ertrinken und nach Wissen dürsten. Seiner Einschätzung nach haben gute Lösungen auf diesem Gebiet ,revolutionäre Auswirkungen auf die gesamte Gesellschaft“. Mehrere andere Teilnehmer geben zu bedenken, dass zu einem beträchtlichen Grad fall- und kontextspezifisch gearbeitet werden muss, um hier zu konkreten Aussagen zu gelangen. Die daraus resultierenden Forschungsergebnisse sind daher oft vergänglich und nur beschränkt reproduzierbar. 
Das Erkenntnisziel „Schaffung verbesserten Wissens über die Architektur von Informationssystemen" kommt auf Rang fünf. Auch hier werden das Argument der Fall- und Kontextspezifität und die daraus resultierenden Probleme der Vergänglichkeit und Beschränktheit als relativierende Faktoren genannt. Im Einklang der Argumentation aus der Analyse des Dreijahreszeitraums kennzeichnet ein weiterer Teilnehmer das vorliegende Erkenntnisziel als „Langfristaufgabe in einer der ureigenen Domänen der Wirtschaftsinformatik. Diese zu vernachlässigen, käme der Forderung gleich, die Medizin solle sich nicht mehr mit der Anatomie beschäftigen.“

\subsection{Vergleich der Ergebnisse für die Drei- und Zehnjahreszeiträume}

Vergleicht man die in beiden Prognosezeiträumen gleichzeitig vorkommenden Erkenntnisziele, so lassen sich hinsichtlich der beiden Cluster „Beherrschung von Komplexität“ und "Neuartigen Anwendungssysteme" zwei signifikante Abweichungen feststellen.

Während das Cluster „Schaffung verbesserten Wissens über die Beherrschung von Komplexität in Informations- und Kommunikationssystemen" im Zehnjahreszeitraum auf Rang eins landet $\left(\overline{\mathrm{x}}_{1,10 \text { Jahre }}=3,70\right)$, wird das gleiche Erkenntnisziel im Dreijahreszeitraum nach der Runde zwei auf Rang neun eingestuft $\left(\overline{\mathbf{X}}_{9,3}\right.$ Jahre $=3,32$; das Signifikanzniveau beträgt $\left.\alpha=0,07\right)$. In den Diskussionsbeiträgen der Teilnehmer finden sich vereinzelt Hinweise, die diese Diskrepanz erklären helfen. Ein Panelist führt hierzu aus, dass die Beherrschbarkeit von Komplexität in Informations- und Kommunikationssystemen eine Frage des dafür notwendigen Aufwands und daher nicht von grundlegender Bedeutung für die Gesellschaft ist. Da sich die Systeme stark voneinander unterscheiden - so derselbe Teilnehmer -, sind konkret anwendbare Lösungen erforderlich. Diese lassen sich jedoch nicht unbedingt reproduzieren. Ein anderer Teilnehmer fügt an, dass ein hohes Maß an Methoden- und Verfahrenskompetenz hinsichtlich einer verbesserten Reproduzierbarkeit von Ergebnissen und höheren Allgemeingültigkeit wünschenswert wäre, bezweifelt aber, dass dieser Anspruch im realen Umfeld erfüllt werden kann.

Die Aussage eines anderen Experten kann demgegenüber stellvertretend für eine Reihe weiterer Beiträge als Gegenposition aufgefasst werden. Sie bringt zum Ausdruck, dass es sich bei der Komplexitätsbeherrschung um ein grundlegendes und dringendes Problem der Praxis handelt, das nur von der Wissenschaft gelöst werden kann. Diese Einschätzung liefert zusammen mit den Argumenten im Zehnjahreszeitraum eine Erklärungshypothese, warum sich die Komplexitätsbeherrschung im Dreijahreszeitraum in der darauffolgenden Runde drei um vier Ränge verbessert hat und warum sie im Zehnjahreszeitraum die Spitzenstellung einnimmt.

Ein ebenfalls signifikanter Unterschied ergibt sich hinsichtlich der „Schaffung verbesserten Wissens über neuartige Anwendungssysteme “ $\left(\overline{\mathrm{x}}_{13,10 \text { Jahre }}=2,85\right.$, Rang dreizehn; $\overline{\mathrm{x}}_{10,3 \text { Jahre }}=$ 3,29 , Rang zehn; $\alpha=0,02$ ). Auch hier wird dem potenziellen Wissensgewinn eine niedrige Allgemeingültigkeit und Reproduzierbarkeit der Ergebnisse gegenüber gestellt. Um zu konkreten Aussagen zu gelangen, muss man nach der Einschätzung eines Experten ,zu einem beträchtlichen Grad fall- und kontextspezifisch“" arbeiten. Die daraus resultierenden Ergebnisse sind oft vergänglich und nur beschränkt reproduzierbar. Im Zehnjahreszeitraums erfolgt eine Akzentuierung dieser Argumentationslinie, da das vorliegende Erkenntnisziel in Verbindung mit einer ,reinen Spezialistenberatung“ gebracht wird. Ein anderer Experte drückt es etwas moderater aus, indem er die Schaffung von Wissen über neuartige Anwendungssysteme als ,domänenspezifische Fragestellung ohne grundlegende Bedeutung für die gesamte Gesell- 
schaft" charakterisiert. Zusammenfassend ist zu interpretieren, dass kurzfristig die Durchdringung neuartiger Anwendungssysteme zu einem vertiefenden Verständnis domänenspezifischer Entwicklungen führen kann, die langfristig nur in Ausnahmefällen verallgemeiner- und reproduzierbar sein dürften.

\section{Diskussion der Ergebnisse}

Wir unterscheiden die inhaltliche Diskussion der Ergebnisse, und zwar zunächst die Betrachtung der als weniger bedeutsam eingeschätzten Erkenntnisziele (Abschnitt 4.1.1) sowie den Vergleich mit den Resultaten der Studie aus dem Jahre 1994 (Abschnitt 4.1.2), gefolgt von methodischen Reflexionen (Abschnitt 4.2).

\subsection{Inhaltliche Diskussion}

\subsubsection{Analyse der als weniger bedeutsam eingeschätzten Erkenntnisziele}

Im Dreijahreszeitraum fällt zunächst die relativ niedrige Bedeutung der Erkenntnisziele „Wissensmanagement“ (Rang sieben), „Management des Wandels von Informationssystemen“ (Rang elf) sowie „Folgenabschätzung neuer Technologien“ (Rang zwölf) auf.

Hinsichtlich der „Schaffung verbesserten Wissens über Wissensmanagement" relativiert die im Panel vertretene Auffassung, es handelt sich um ein reines Modethema, die Bedeutung dieses Erkenntnisziels. Einige Teilnehmer versuchen, dieser Einschätzung entgegenzutreten. Ein Experte sieht das Wissensmanagement zwar ein Modethema der Praktikergazetten ist, fügt aber an, dass wenn ein Thema für soviel Furore in der Praxis sorgt, dann sollte man sich wissenschaftlich damit befassen. In den Augen eines anderen Teilnehmers hat die Wirtschaftsinformatik einiges geleistet, wenn sie beim Wissensmanagement nicht nur mit Begriffen um sich wirft, sondern echte Lösungen findet.

Zu dem Erkenntnisziel „Schaffung verbesserten Wissens über das Management des Wandels von Informationssystemen" finden sich nur vereinzelt Anhaltspunkte über die niedrige Einstufung. Ein Teilnehmer merkt hierzu an, dass es sich um eine hochpraktische Aufgabe handelt, die sich jedoch stark auf Einzelfälle beschränkt und abhängig von der eingesetzten Technologie ist. Der Auffassung eines anderen Experten zufolge sind die fallspezifischen Erkenntnisse hierzu im Allgemeinen meist schwer anwendbar. Noch drastischer drückt ein weiterer Panelist aus, dass das Thema so allgemein ist, dass es kaum Relevanz gewinnen kann.

Für die niedrige Bewertung des Clusters ,Schaffung verbesserten Wissens über die Folgenabschätzung neuer Technologien" finden sich ebenfalls in der qualitativen Auseinandersetzung einige Hinweise. Ein Teilnehmer merkt hierzu an, dass Aussagen zur Folgenabschätzung schwer möglich sind und durch die Realität immer eingeholt werden. In den Augen anderer Teilnehmer ist die Zukunft blind. Es ist daher vermessen, die Erarbeitung prognostischer, fallweiser und inhärent fehlerbehafteter Aussagen in einem derart schnelllebigen Geschäft zu einer Kernkompetenz zu erheben.

Im Zehnjahreszeitraum fallen die Cluster „Human Resource Management in der Informationstechnik“ (Rang elf), „Grundlagen der Wirtschaftinformatik“ (Rang dreizehn) und „Schnittstellen der Wirtschaftsinformatik zu anderen Disziplinen“ (Rang fünfzehn) auf; die beiden Letztgenannten liegen auch auf den hinteren Plätzen im Dreijahreszeitraum (,Grundlagen“" auf Rang vierzehn und „Schnittstellen“ auf Rang dreizehn). 
Die „Schaffung verbesserten Wissens über Human Resource Management in der IT“ wird von mehreren Teilnehmern als wichtiger Faktor in der aktuellen Arbeitsmarktdiskussion gesehen. Dagegen findet sich in der qualitativen Diskussion ein prägnanter Hinweis über eine niedrigere Bewertung dieses Erkenntnisziels. In den Augen eines Experten handelt es sich um ein Randthema, das eher der BWL zugeordnet werden kann. Human Resource Management ist nicht IT-spezifisch und stellt für jede neue Technologie eine Problemfacette dar.

Über die Bedeutung der „Schaffung verbesserten Wissens über Grundlagen der Wirtschaftsinformatik" gibt ein Teilnehmer nachdrücklich zu bedenken, dass es sich bei diesem Erkenntnisziel um Selbstverständlichkeiten einer Wissenschaftsdisziplin handelt. Wenn hier ein Handlungsbedarf existiert, so derselbe Panelist, dann hat die Wirtschaftsinformatik ein Problem.

Hinsichtlich der „Schaffung verbesserten Wissens über Schnittstellen der Wirtschaftsinformatik" akzentuieren einige Teilnehmer die niedrige Bedeutung für die Gesellschaft und die geringe Anwendbarkeit in der Praxis. Ein Teilnehmer charakterisiert die Abgrenzung zu anderen Wissenschaften als ein akademisches Problem, das den Praktiker wenig interessiert. Ein anderer Teilnehmer spricht in diesem Kontext von einer „Nabelschau, die für Außenstehende von begrenztem Interesse sein dürfte“.

\subsubsection{Vergleich mit den Ergebnissen von 1994}

Trotz der eingangs aufgezeigten Unterschiede im Bezugsrahmen sowie der aus dem offenen Frageformat in Runde eins resultierenden semantischen Abweichungen soll ein Vergleich der Studien von 1999 und 1994 vorgenommen werden. Für einen Prognosezeitraum von zehn Jahren wird untersucht,

- ob und wie sich die wichtigsten Erkenntnisgegenstände von 1994 in der Studie von 1999 wiederfinden und

- ob und wie die wichtigsten Erkenntnisziele von 1999 bereits in der Studie von 1994 Eingang gefunden hatten.

Gemäß den Ergebnissen der Studie von 1994 sollte - so die Aussage auf Rang eins - die Ausrichtung der Wirtschaftsinformatik als Wissenschaft mit starkem Bezug zur Organisationslehre erfolgen, die versucht, den Aufbau und die Abläufe sozio-technischer Systeme zu beschreiben und zu optimieren (vgl. hierzu und im Folgenden [KHP95, 560 ff.]). Es handelt sich hierbei um ein komplexes und umfassendes Konstrukt, das in drei Ebenen unterteilt wurde:

- die Individualebene,

- die Arbeitsgruppen-, Abteilungs- und Unternehmensebene sowie

- die Markt- und Gesellschaftsebene.

Im Zusammenhang mit der Ausrichtung der Wirtschaftsinformatik als Wissenschaft mit starkem Bezug zur Organisationslehre auf der Individualebene fällt auf, dass die beiden wichtigsten Nennungen von 1994, nämlich die Untersuchung der Arbeitsteilung zwischen Mensch und Maschine und die Untersuchung der Mensch-Maschine-Schnittstelle, in den Ergebnissen des Zehnjahreszeitraums der Studie im Jahr 1999 ebenfalls als bedeutsam eingestuft werden. Das Erkenntnisziel der Gestaltung von Mensch-Maschine-Schnittstellen in Verbindung mit der Schaffung verbesserten Wissens über Anwender kommt in der vorliegenden Studie auf Rang drei und wurde bereits weiter oben erörtert. Die Schaffung verbesserten Wissens über 
neue Arbeitsteilungen und Formen der Kollaboration zwischen verschiedenen Akteuren landet auf Rang sechs. In diesem Erkenntnisziel wird von einigen Panelisten der Kern der zukünftigen Organisation der Gesellschaft gesehen. Dahinter verbirgt sich die Frage, ob sich die Grundstruktur der Arbeits- und Unternehmenswelt durch Informations- und Kommunikationstechnologien ändert. Dass dieses Erkenntnisziel nicht (noch) höher eingestuft wurde, kann vor dem Hinweis eines anderen Teilnehmers betrachtet werden, der dieses Thema als ,,abgegrast" bezeichnet.

Auf der Arbeitsgruppen-, Abteilungs- und Unternehmensebene wurden 1994 die Beschreibung und Optimierung von Geschäftsprozessen sowie die Wechselwirkungen von Informations- und Kommunikationstechnik und der Unternehmensorganisation am wichtigsten eingestuft. Das zu Beginn der 90er Jahre häufig betrachtete Thema „Business Process Reengineering“ findet im Rahmen der vorliegenden Studie lediglich im Kontext der Architektur von Informationssystemen eine kurze Erwähnung. Damit kommt zum Ausdruck, dass Geschäftsprozesse eine relevante Ebene repräsentieren bzw. Sicht auf Informationssysteme darstellen. Die Wechselwirkungen der Technik mit der Unternehmensorganisation wird in der aktuellen Studie vor dem Hintergrund innovativer Organisationsformen im Zusammenhang mit neuen Arbeitsteilungen und Formen der Kollaboration zwischen Akteuren behandelt und, wie bereits im letzten Abschnitt angedeutet, auf Rang sechs eingestuft. Zudem haben die Experten den Zusammenhang zwischen Informationstechnologien und Organisation im Dreijahreszeitraum als eigenständiges Cluster behandelt, das auf Rang drei landete (und ebenfalls bereits erörtert wurde).

Die Experten erachteten 1994 auf der Markt- und Gesellschaftsebene interorganisatorische Informationssysteme, virtuelle Unternehmen und elektronische Märkte als bedeutsam. Während interorganisatorische Informationssysteme in der vorliegenden Studie nur am Rande bei der Diskussion über die Architektur von Informationssystemen (Rang fünf) aufgegriffen werden, sind Netzmärkte und virtuelle Märkte im Zehnjahreszeitraum von großer Bedeutung, was sich auch am zweiten Rangplatz wiederspiegelt. Insofern lassen sich auch hier gewisse Fortschreibungstendenzen aus der Untersuchung von 1994 erkennen.

Im Jahr 1994 eroberte die Ausrichtung der Wirtschaftsinformatik als funktionale Betriebswirtschaftslehre, die vornehmlich die Funktion der betrieblichen Informationsverarbeitung im Unternehmen erforscht, den zweiten Rang. Darunter wurden das Management der betrieblichen Informationsverarbeitung, Instrumente zu deren Planung, Steuerung und Kontrolle sowie das Instrument zur Messung des ökonomischen Beitrags computergestützter Anwendungssysteme subsummiert. In der vorliegenden Studie kommen Erkenntnisziele dieser Art nur am Rande vor. Lediglich im Cluster Informationsmanagement / Wissensmanagement (Rang vier) wird die Frage formuliert, wie sich virtuelle Unternehmen und Informationstechnik-basierte Unternehmensnetzwerke auf die Aufgaben des IV-Managements auswirken werden.

Vor fünf Jahren diskutierten die Experten die Wirtschaftsinformatik zudem in der Ausrichtung einer Informationswissenschaft (Rang drei), die versucht, die Ökonomie des Leistungsfaktors Information und seine gezielte Bereitstellung zu erforschen. In der im vergangenen Jahr durchgeführten Studie wird dieser Forschungsgegenstand im Rahmen des Clusters Informationsmanagement / Wissensmanagement nur am Rande des Zehnjahreszeitraums behandelt. Jedoch kommt im Dreijahreszeitrum das Erkenntnisziel „Information als Produkt“ auf Rang vier. Wie bereits oben aufgezeigt wird, spielt diese Zielsetzung in einer sich formierenden Informations- bzw. Wissensgesellschaft eine herausragende Rolle. Warum sie im Zehnjahreszeitraum keine Berücksichtigung findet, kann allenfalls mit dem Hinweis eines Teil- 
nehmers erklärt werden, der davon ausgeht, dass diese Thematik bereits facettenreich von den Informationswissenschaften bearbeitet worden ist.

Untersucht man, ob und welche Bedeutung bestimmte Erkenntnisziele der vorliegenden Studie vor fünf Jahren hatten, so fallen die Cluster „Beherrschung der Komplexität in Informations- und Kommunikationssystemen “ und die „, Schaffung verbesserten Wissens über die Architektur von Informationssystemen " auf. Das erstgenannte Erkenntnisziel fand 1994 keine explizite Berücksichtigung. Insofern kann man die in der vorliegenden Studie durchgeführte Komplexitätsdiskussion als Ausdruck der immer stärkeren Durchdringung von Informationsund Kommunikationstechnologien in Unternehmen und Gesellschaft sowie den damit verbundenen Problemen ihrer Beherrschbarkeit und Folgenabschätzung aufgrund veränderter Arbeitsteilungen interpretieren. Dagegen wurde die Ausrichtung der Wirtschaftsinformatik als Lehre der Beschreibung von Architekturen in der damaligen Studie zwar berücksichtigt, jedoch mit dem achten Rang als weniger bedeutend eingeschätzt. Dabei erörterten die Experten 1994 insbesondere die Modellierung als Prozess der Architekturentwicklung, die Entwicklung von Methoden und Werkzeugen zur Architekturentwicklung und die Behandlung der Datenintegration als speziellen Architekturausschnitt. Dagegen drehen sich die Architekturdiskussionen in der aktuellen Studie um Komponentenarchitekturen und Referenzmodelle, die Schaffung einer Konstruktionslehre für Informationssysteme sowie die Bereitstellung von Methoden zur Integration neuer Technologien in existierende DV-Architekturen. Dies lässt darauf schließen, dass die Frage der Architektur noch nicht abschließend gelöst und von den damit abzubildenden betriebswirtschaftlichen Aufgaben abhängig ist. Die Bedeutung von Architekturfragen nimmt offenbar aufgrund der zunehmenden Verbreitung von Informationsund Kommunikationstechnologien zu.

\subsection{Methodische Diskussion}

Als Hilfsgrößen für die Validität und Reliabilität der Studie sollen drei Indikatoren herangezogen werden: die Abbruchrate der Teilnehmer, die Komponentenladungen der Konstituenten und die Ergebnisse einer im Anschluss an die Delphi-Studie durchgeführten Zusatzbefragung (,Supplement Study“).

Bereits oben wurde ausgeführt, dass während der Studie insgesamt fünf Teilnehmer den Delphi-Prozess nicht bis zum Ende fortgesetzt haben. Dies entspricht einer Abbruchquote von 14\%. Im Vergleich zur Untersuchung von 1994 und zu anderen Delphi-Studien ist diese Quote gering [KHP95; Hein96] und wir können davon ausgehen, dass die Abbrecher die Ergebnisse nicht signifikant beeinflussten. 


\begin{tabular}{|l|c|c|c|}
\hline & \multicolumn{3}{|c|}{ Komponente } \\
\cline { 2 - 4 } & 1 & 2 & 3 \\
\hline Grundlegende Bedeutung &,- 723 & & \\
Anwendbarkeit in der Praxis &, 944 & &, 991 \\
Allgemeingültigkeit der Aussagen & &, 985 & \\
Reproduzierbarkeit von Ergebnissen & & & \\
\hline
\end{tabular}

Extraktionsmethode: Hauptkomponentenanalyse

Rotationsmethode: Varimax mit Kaiser-Normalisierung

Tabelle 3 Komponentenladungen der Konstituenten (Rotierte Komponentenmatrix)

Wir haben mithilfe einer Hauptkomponentenanalyse die Validität der verwendeten Konstituenten untersucht [BEPS00]. Diese erwiesen sich bis auf eine Ausnahme (aus vier Faktoren) als valide. Lediglich die Konstituenten „Grundlegende Bedeutung für die Gesellschaft" und „Anwendbarkeit der zu erwartenden Ergebnisse in der Praxis“ laden auf jeweils eine gemeinsame Komponente. Dabei weist die erstgenannte Konstituente ein negatives Vorzeichen, die zweitgenannte ein positives Vorzeichen auf, was auf einen negativen statistischen Zusammenhang dieser beiden Größen schließen lässt. Damit drückt das vorliegende Datenmaterial folgende Beziehung aus: Je höher die grundlegende Bedeutung eines Erkenntnisziels für die Gesellschaft, desto niedriger ist die Anwendbarkeit dieses Ziels in der Praxis und umgekehrt. Insofern kann man bei zukünftigen Untersuchungen die Anzahl der Konstituenten auf drei reduzieren. Die vorliegende Hauptkomponentenanalyse erklärt über 99\% der Gesamtvarianz.

In der Untersuchung von 1994 wurden zwei Probleme konstatiert, die im Rahmen der vorliegenden Studie mithilfe einer Zusatzbefragung genauer untersucht werden sollten. Zum einen treten in Delphi-Studien beim Übergang vom offenen in ein geschlossenes Frageformat Informationsverluste bzw. semantische Unschärfen infolge des gewählten Klassifikationsschemas (hier: „Cluster“) auf. Zum anderen bieten Delphi-Studien die Möglichkeit zum anonymen Diskurs mit anderen Teilnehmern, d. h. der argumentativen Bezugnahme der Teilnehmer untereinander. 1994 beklagten Teilnehmer ein zu geringes Eingehen auf vorliegende Aussagen von Experten. Wie stellt sich diese Situation 1999 dar?

An der Zusatzbefragung nahmen 23 von 30 Experten, die bis zum Ende der dritten Runde mitwirkten, also $76 \%$ teil. Die Bewertung erfolgte auf der Basis von Schulnoten $(1=\mathrm{sehr}$ gut, ..., 6 = ungenügend). Das offene Frageformat in Runde eins wird von den Teilnehmern einhellig begrüßt (Durchschnittsnote 1,70). Zum Teil noch bessere Bewertungen erhält das Moderatorenteam für seine Neutralität $(1,61)$, seine Unterstützung bei technischen und/oder inhaltlichen Problemen bzw. Fragen $(1,60)$ und die Durchführung der Studie über das Internet $(1,73)$. Etwas schlechtere, aber immer noch gute bis zufriedenstellende Zensuren gibt es für die Qualität der Clusterbildung $(2,45)$. Folgende qualitativen Argumente sind in diesem Zusammenhang zu nennen:

- Ein Teilnehmer merkt an, dass die Cluster hierarchisch im Sinne von „topical areas“ gebildet wurden, aber vernetzt sind, was seiner Ansicht nach legitim ist. Diese „Vernetzung“ lässt sich mithilfe von Korrelationsmatrizen bzw. Faktorenanalysen nachweisen und wird Gegenstand eines weiteren Beitrags sein.

- Ein anderer Experte gibt zu bedenken, dass die Clusterbildung zu einem Zeitpunkt erfolgte, an dem er eigentlich nicht „so weit war“. Er fügt hinzu, dass er jedoch im weiteren Verlauf der Studie mit der entstandenen Struktur gut umgehen konnte. 
- Ein anderer Panelist merkt an, dass die (gleichartigen) Erkenntnisziele im Drei- und Zehnjahreszeitraum hätten gleich benannt werden können. Auch aus der Sicht der Autoren wäre dies wünschenswert gewesen, da man so eine höhere Vergleichbarkeit der unterschiedlichen Prognosezeiträume erreicht hätte. Insofern spricht das Widerstehen gegen eine solche Vereinfachung für im Verlauf der Studie dokumentierte semantische Unterschiede in den artikulierten Erkenntniszielen.

- Ein weiterer Teilnehmer merkt an, dass die Cluster naturgemäß allgemein gehalten sind und jeder Panelist etwas anderes darunter versteht. Die Autoren dieser Studie waren und sind sich dieser Problematik bewusst. Daher wurden im Gegensatz zu 1994 die hierarchische Klassifikationsstruktur und die zu jedem Cluster zusammengefassten Aussagen bereits während der Studie vollständig offen gelegt, um semantische Unschärfen und unterschiedliche Interpretationsmuster zu reduzieren.

- Ein anderer Experte vertritt die Ansicht, dass die Herauslösung der Interdisziplinarität nach Runde zwei die Verbindung der Runden zwei und drei beeinträchtigt. Dem kann zunächst entgegnet werden, dass die Interdisziplinarität zu Beginn der Studie als unwichtigste Konstituente eingestuft wurde. Trotzdem muss eingeräumt werden, dass sich bei einer Ausklammerung der Interdisziplinarität einige Ränge geringfügig verändern. Die im Rahmen dieses Beitrags vorgenommenen Rangbildungen haben den Einfluss der Interdisziplinarität jedoch vollständig ausgeklammert, um gerade die geforderte Verbindung wiederherzustellen.

Nur zufriedenstellend bewerten die Teilnehmer ihre Bezugnahme auf vorliegende Argumente $(2,81)$. Hierzu kommentierten einzelne Experten, dass dies eine Aufwandsfrage ist, gerade vor dem Hintergrund, dass die gesamten Panel-Daten im Internet zur Verfügung standen bzw. die Bearbeitungsfristen für eine stärkere Bezugnahme zu kurz gewesen sind. Insofern ist es kaum verwunderlich, dass den eingeräumten Bearbeitungsfristen (jeweils vierzehn Tage in allen drei Runden) die schlechteste Durchschnittsnote in der Zusatzbefragung eingeräumt wird $(3,09)$, obwohl die im Anschreiben vorgenommene Schätzung des zur Beantwortung benötigten Zeitaufwands besser abschneidet $(2,59)$. Ein Teilnehmer gibt zu bedenken, dass daran nicht die Moderatoren schuld sind, sondern die ständige Überlastung mit nicht-wissenschaftlichen Aufgaben im Hochschulbetrieb. Trotzdem sollte in Erwägung gezogen werden, die Bearbeitungsfristen bei der nächsten Studie zu verlängern.

Der Nutzen der durchgeführten Studie für den Fortschritt der Wirtschaftsinformatik als Wissenschaft wurde mit noch gut bewertet $(2,29)$. Ein ähnliches Bild ergibt sich bei der Einschätzung des Nutzens der Untersuchung für die persönliche Arbeit der Teilnehmer (2,3). Insgesamt signalisieren 22 von 23 Teilnehmern der Zusatzbefragung ihre Bereitschaft, an einer Wiederauflage der Studie in fünf Jahren teilzunehmen. Die guten, an zwei Stellen befriedigenden Noten lassen die Schlussfolgerung zu, dass die Qualität der Untersuchung als akzeptabel eingestuft werden kann, was sich auch in der relativ niedrigen Abbruchrate ausdrückt. Darüber hinaus liefert die Zusatzbefragung wertvolle Hinweise für zukünftige Studien dieser Art.

\section{Zusammenfassung und Ausblick}

Die wichtigsten Ergebnisse der zweiten, im Jahr 1999 durchgeführten Delphi-Studie, die nach den zentralen Erkenntniszielen der Wirtschaftsinformatik fragte, damit diese sich im Wettbewerb mit den Nachbardisziplinen Betriebswirtschaftslehre und Informatik behaupten kann, lassen sich wie folgt zusammenfassen: 
- Für den Zeitraum der nächsten drei Jahre haben 30 Experten die Schaffung verbesserten Wissens über

- Netzmärkte und E-Commerce,

- die Architektur von Informations- und Kommunikationssystemen sowie

- den Zusammenhang zwischen Informationstechnologien und Organisation

als bedeutendste Erkenntnisziele formuliert.

- Im Zehnjahreszeitraum wird der Schaffung verbesserten Wissens über

- die Beherrschung von Komplexität in Informations- und Kommunikationssystemen,

- Netzmärkte und virtuelle Märkte sowie

- die Gestaltung der Anwender bzw. Mensch/Maschine-Schnittstelle

die größte Bedeutung beigemessen.

- Die Schaffungen verbesserten Wissens über Grundlagen der Wirtschaftsinformatik sowie über Schnittstellen zu anderen Wissenschaften landen in beiden Prognosezeiträumen auf den letzten Plätzen.

- Das Panel sieht die Anwendbarkeit von Forschungsergebnissen in der Praxis als wichtigste Einflussgröße für die Beurteilung von Erkenntniszielen der Wirtschaftsinformatik im Wettbewerbskontext zu den Nachbardisziplinen an.

- $90 \%$ der Experten sehen das interdisziplinäre Arbeiten als eine Kernkompetenz der Wirtschaftsinformatik und fast drei Viertel von ihnen plädieren für eine Vertiefung der interdisziplinären Bezüge zwischen den Wirtschaftswissenschaften und der Informatik.

- Eine Zusatzbefragung nach Abschluss der drei Delphi-Runden erbrachte Hinweise über die Validität der durchgeführten Studie und wertvolle Anregungen für zukünftige Vorhaben dieser Art.

Das in der Ausgangskonzeption dieser Studie zugrunde gelegte Wettbewerbsmodell muss allerdings kritisch in Frage gestellt werden. Die Ergebnisse im Zusammenhang mit der Interdisziplinarität lassen vielmehr darauf schließen, dass „Coopetition“ das Verhältnis mit den Nachbardisziplinen kennzeichnet [NaBr99; ShVa99]. Demzufolge steht die Wirtschaftsinformatik prinzipiell mit der Betriebswirtschaftslehre und der Informatik in Konkurrenz, kooperiert aber gleichzeitig zunehmend mit diesen Disziplinen. Neben Tagungen und Veröffentlichungen, die von Wirtschaftsinformatikern gemeinsam mit Informatikern und Wirtschaftswissenschaftlern abgehalten bzw. geschrieben werden, kommt dies auch im aktuellen Schwerpunktprogramm der Deutschen Forschungsgemeinschaft „Intelligente Agenten und betriebswirtschaftliche Anwendungsszenarien“ zum Ausdruck. Kooperatives Verhalten von Konkurrenten erscheint vor allem dann sinnvoll, wenn die Spezialisierungsvorteile der betreffenden Disziplinen (z. B. Domänenwissen und Praxisbezug versus Technologiewissen und Grundlagenbezug) so gebündelt werden, dass dadurch Wettbewerbsvorteile im Bereich von Schlüsseltechnologien (hier: Informations- und Kommunikationstechnologien) im Sinne einer Stärkung der Innovationskraft sowie Wettbewerbsvorteile gegenüber anderen Disziplinen (z. B. Natur- oder Kulturwissenschaften) im Sinne einer Ressourcensicherung entstehen.

Gelingt es der Wirtschaftsinformatik, bei Wahrung ihres anwendungsbezogenes Profils die Verknüpfung von betriebswirtschaftlichen und informationstechnologischen Problemstellungen schneller, tiefgreifender und verwertbarer zu vollziehen als ihre Nachbardisziplinen, dürfte sie auch zukünftig ein begehrter Kooperationspartner der Praxis und von anderen Wissenschaftsdisziplinen bleiben. 


\section{Literatur}

[BaCo96] Bamberg, G.; Coenenberg, A.G.: Betriebswirtschaftliche Entscheidungslehre, 9. Auflage. München 1996.

[BEPS00] Backhaus, K.; Erichson, B.; Plinke, W.; Schuchard-Fischer, C.; Weiber, R.: Multivariate Analysemethoden: eine anwendungsorientierte Einführung. Berlin 2000.

[Hein96] Heinzl, A.: Die Evolution der betrieblichen DV-Abteilung: Eine lebenszyklustheoretische Analyse. Heidelberg 1996.

[KHP95] König, W.; Heinzl, A.; von Poblotzki, A.: Die zentralen Forschungsgegenstände der Wirtschaftsinformatik in den nächsten zehn Jahren. In: WIRTSCHAFTSINFORMATIK, Heft 6/1995, S. 558-569.

[KHRP96] König, W.; Heinzl, A.; Rumpf, M.-J.; von Poblotzki, A.: Zur Entwicklung der Forschungsmethoden und Theoriekerne der Wirtschaftsinformatik. Eine kombinierte Delphi- und AHP-Untersuchung. In: Heilmann, H.; Heinrich, L.J.; Roithmayr, F. (Hrsg.): Information Engineering. München und Wien 1996, S. 35-65.

[NaBr99] Nalebuff, B.; Brandenburger, A.: Coopetition - Kooperativ konkurrieren. Frankfurt 1996.

[RoWr99] Rowe, G.; Wright, G.: The Delphi technique as a forecasting tool: issues and analysis. In: International Journal of Forecasting 15 (1999), S. $353-375$.

[Sack75]

Sackmann, H.E.: Delphi critique. Lexington, Ma. 1975.

[ShVa99]

Shapiro, C.; Varian, H.: Information rules. Boston, Ma. 1999.

\section{Anhang A: Liste der 30 bis zum Schluss der Runde drei aktiven Mitglieder des Panel}

$\begin{array}{lll}\text { Prof. Dr. } & \text { Hans-Jürgen } & \text { Appelrath } \\ \text { Prof. Dr. } & \text { Jörg } & \text { Becker } \\ \text { Prof. Dr. } & \text { Rainer } & \text { Bischoff } \\ \text { Prof. Dr. } & \text { Hans Ulrich } & \text { Buhl } \\ \text { Prof. Dr. } & \text { Georg } & \text { Disterer } \\ \text { Dr. } & \text { Klaus } & \text { Eierhoff } \\ \text { Prof. Dr. } & \text { Eberhard } & \text { Feess } \\ \text { Prof. Dr. } & \text { Wolfgang } & \text { Glatthaar } \\ \text { PD Dr. } & \text { Rainer } & \text { Göhring } \\ \text { Prof. Dr. Joachim } & \text { Griese } \\ \text { Prof. Dr. } & \text { Oliver } & \text { Günther } \\ \text { Prof. Dr. } & \text { Hans Robert } & \text { Hansen } \\ \text { Prof. Dr. } & \text { Ulrich } & \text { Hasenkamp } \\ \text { Prof. Dr. } & \text { Otthein } & \text { Herzog } \\ \text { Prof. Dr. } & \text { Matthias } & \text { Jarke } \\ \text { Prof. Dr. } & \text { Stefan } & \text { Kirn }\end{array}$

Universität Oldenburg
Universität Münster
Fachhochschule Furtwangen
Universität Augsburg
Fachhochschule Hannover
Bertelsmann AG, Gütersloh
Universität Frankfurt a. M.
DG Bank, Frankfurt
Dr. Göhring und Partner Unternehmensberatung AG, Wiesbaden
Universität Bern
Humboldt-Universität zu Berlin
Wirtschaftsuniversität Wien
Universität Marburg
Universität Bremen
RW Technische Hochschule Aachen
Technische Universität IImenau




\begin{tabular}{|c|c|c|c|}
\hline Dr. & Lutz & Kolbe & Deutsche Bank AG, Eschborn \\
\hline Prof. Dr. & Jan-Pieter & Krahnen & Universität Frankfurt a. M. \\
\hline Prof. Dr. & Hermann & Krallmann & Technische Universität Berlin \\
\hline Prof. Dr. & Helmut & Krcmar & Universität Hohenheim \\
\hline Prof. Dr. & Peter & Lockemann & Universität Karlsruhe \\
\hline Prof. Dr. & Günter & Müller & Universität Freiburg \\
\hline Prof. Dr. & Andreas & Oberweis & Universität Frankfurt a. M. \\
\hline Prof. Dr. & Hubert & Österle & Universität St. Gallen \\
\hline Prof. Dr. & Arnold & Picot & Universität München \\
\hline Prof. Dr. & Claus & Rautenstrauch & Universität Magdeburg \\
\hline Prof. Dr. & August-Wilhelm & Scheer & Universität Saarbrücken \\
\hline Prof. Dr. & Elmar & $\operatorname{Sin} z$ & Universität Bamberg \\
\hline Prof. Dr. & Christof & Weinhardt & Universität Gießen \\
\hline Prof. Dr. & Stephan & Zelewski & Universität GH Essen \\
\hline
\end{tabular}

\section{Anhang B: Liste der vollständigen Cluster-Titel}

Name des Clusters im Dreijahreszeitraum (in Rangfolge)

3h) Verbessertes Wissen über Netzmärkte und E-Commerce schaffen

3b) Verbessertes Wissen über Architektur von Informations- und Kommunikationssystemen schaffen

3n) Verbessertes Wissen über den Zusammenhang zwischen Informationstechnik und Organisation schaffen

3f) Verbessertes Wissen über Information als Produkt schaffen

3a) Verbessertes Wissen über neue Techniken der Wirtschaftsinformatik schaffen

31) Verbessertes Wissen über Wirtschaftlichkeit und Produktivität schaffen

3k) Verbessertes Wissen über strategische Wirkungen von Informationssystemen / Innovationen durch Informationssysteme schaffen

3m) Verbessertes Wissen über Wissensmanagement schaffen

3c) Verbessertes Wissen über Beherrschbarkeit von Komplexität in Informations- und Kommunikationssystemen schaffen

3i) Verbessertes Wissen über neuartige Anwendungssysteme schaffen

3g) Verbessertes Wissen über Management des Wandels von Informationssystemen schaffen

3d) Verbessertes Wissen über Folgenabschätzung neuer Technologien schaffen

3j) Verbessertes Wissen über Schnittstellen der Wirtschaftsinformatik zu anderen Wissenschaften schaffen

3e) Verbessertes Wissen über Grundlagen der Wirtschaftsinformatik schaffen

Name des Clusters im Zehnjahreszeitraum (in Rangfolge)

10c) Verbessertes Wissen über Beherrschung von Komplexität in Informations- und Kommunikationssystemen schaffen

10k) Verbessertes Wissen über Netzmärkte und virtuelle Märkte schaffen

10a) Verbessertes Wissen über Anwender- / Mensch-Maschine-Schnittstellen schaffen

10g) Verbessertes Wissen über Informationsmanagement / Wissensmanagement schaffen

10b) Verbessertes Wissen über Architektur von Informationssystemen schaffen

10m) Verbessertes Wissen über neue Arbeitsteilungen und Formen von Kollaboration zwischen verschiedenen Akteuren schaffen

10n) Verbessertes Wissen über neue Lehr- und Lernformen schaffen

10h) Verbessertes Wissen über Künstliche-Intelligenz-Systeme / menschenähnliche Systeme schaffen

10i) Verbessertes Wissen über gesellschaftliche Folgen des Einsatzes von Informationssystemen schaffen

10o) Verbessertes Wissen über wertortientierte Unternehmensführung und Beitrag der Informations- und Kommunikationssysteme schaffen

10f) Verbessertes Wissen über Human Resource Management in der Informationstechnik schaffen

10j) Verbessertes Wissen über Management des Wandels von Informationssystemen sowie anderer Objekte schaffen

10I) Verbessertes Wissen über neuartige Anwendungssysteme schaffen

10e) Verbessertes Wissen über Grundlagen der Wirtschaftsinformatik schaffen

10d) Verbessertes Wissen über Schnittstellen der Wirtschaftsinformatik schaffen 
Which future research subjects shall be persued by the German speaking IS community (Wirtschaftsinformatik) in order to maintain competitive against other disciplines like business

administration and computer science? This article summarizes the major results of a Delphi study which was conducted in 1999 in order to answer this question drawing on the expertise of leading academics and practitioners in the field. Thus, the foundations of this study will be outlined as well as its methodology, the main findings, and a comparison to a similar study which was conducted in 1994. 